\title{
GCND per il pianeta. Un forte invito ad agire per tutti: medici, infermieri e pazienti
}

\author{
Marco Lombardi ${ }^{1}$, Franco Bergesio \\ ${ }^{1}$ Editor in Chief, Giornale di Clinica Nefrologica e Dialisi, Firenze - Italy \\ ${ }^{2}$ Section Editor, GCND per il pianeta, Firenze - Italy
}

Carissimi lettori,

lo scorso anno abbiamo ambiziosamente lanciato un progetto rivolto al coinvolgimento del mondo sanitario nella salvaguardia del nostro bene comune il pianeta. Questo progetto iniziale, che è stato commentato da tanti lettori attraverso una survey dedicata, è cresciuto con nostra grande soddisfazione. C'è ancora molta strada da fare, ma quello spazio che un anno fa sembrava una scommessa, GCND per il pianeta, è adesso diventato una realtà pronta a crescere e ad arricchirsi di informazioni, esperienze e contributi direttamente inviati anche da voi lettori.

Il Board di questa sezione, coordinato da Franco Bergesio, è formato da 4 medici (3 nefrologi ed un 1 ematologo), tutti iscritti a ISDE (International Society of Doctors for Environment) un'associazione internazionale di medici (ma non solo) che studiano le conseguenze sulla nostra salute dei cambiamenti climatici e dell'inquinamento ambientale, e e dal Prof Roberto Buizza, fisico esperto in ambiente dell'Istituto Superiore Sant'Anna di Pisa. Se il Prof. Buizza ha firmato l'articolo a conclusione del 2020 per la sezione (1), sarà Roberto Romizi, Presidente italiano ISDE, a firmare il primo articolo 2021.

Quanto accaduto lo scorso anno ci ha convinti che è necessario aumentare l'impegno alla conservazione del nostro pianeta (che tra l'altro è l'unico che abbiamo!). È necessario coinvolgere l'intero mondo sanitario, a partire dai medici ma allargandolo anche agli infermieri e a tutti gli addetti all'assistenza e cura dei pazienti, inclusi non ultimi i pazienti stessi.

L'obiettivo della sezione non sarà solo l'informazione tramite articoli scientifici dedicati ma anche, e soprattutto, quello di aprire un dialogo con i lettori che vorranno dare il

Received: January 17, 2021

Accepted: January 18, 2021

Published online: February 18, 2021

Indirizzo per la corrispondenza:

Marco Lombardi

Ospedale del Mugello

Via Della Resistenza, 60

50032 Borgo San Lorenzo, Firenze - Italy

lombardim@tin.it loro contributo con commenti, idee o suggerimenti affinché i medici si sentano co-responsabili di quanto viene - o non viene - messo in opera per salvaguardare il nostro ambiente e dunque la nostra salute.

In particolare, la sezione vorrà anche essere un'opportunità per affrontare e contribuire a diffondere i temi della "green nephrology", facilitando la transizione verso una nefrologia più ecologica. Saranno perciò benvenute le esperienze che i lettori ci vorranno riportare a riguardo.

Tra le novità sono previste interviste a personaggi di primo piano della nefrologia italiana con esperienze rilevanti da condividere e brevi survey mirate.

L'esigenza di coinvolgere il mondo medico nella tutela dell'ambiente e la difesa dalle malattie conseguenti al cambiamento climatico, a partire dalle nostre società scientifiche, nasce alla fine del 2019, in epoca pre-COVID. L'esperienza della pandemia ha purtroppo confermato i pericoli connessi con l'attuale modello di sviluppo e ha ulteriormente rafforzato questa esigenza, come dimostrato anche dalla survey organizzata dal nostro giornale (2).

L'anno appena passato verrà probabilmente ricordato come "annus horribilis" $(3,4)$. Nel corso del 2020 sono state trovate nella placenta di esseri umani sani tracce di microplastiche (5): "In total, 12 micro plastic fragments (ranging from 5 to $10 \mu \mathrm{m}$ in size), with spheric or irregular shape were found in 4 placentas ( 5 in the fetal side, 4 in the maternal side and 3 in the chorioamniotic membranes)" il che significa la creazione di bambini-cyborg, ovvero composti non solamente di cellule umane, ma da una 'mistura' di componente biologica e di componente inorganica di plastiche (anche di propilene ovvero, ad esempio, il materiale delle nostre bottiglie e tappi di plastica) (5).

Ma il 2020 sarà ricordato anche per essere l'anno in cui la cosiddetta massa antropica (quella creata dall'uomo o human-made mass) ha superato la biomassa vivente (animale e vegetale): solamente gli oggetti in plastica che abbiamo prodotto pesano il doppio di tutti gli animali del mondo! La stima, pubblicata su Nature (6) per L'Istituto Israeliano per le Scienze, riporta che i 1.100 miliardi di tonnellate di massa antropica superano i 1.000 miliardi di tonnellate di biomassa. Sono dati in parte già paventati nel 2018 quando su Proceedings of the National Academy of 
Sciences (PNAS) (7) si scriveva che l'umanità in termini di peso rappresenta lo $0,01 \%$ degli esseri viventi ma al contempo incide incredibilmente di più sugli equilibri dell'eco sistema del pianeta.

II 2020 ha molti tristi primati certo, tuttavia vogliamo sottolinearne uno positivo. Ha rappresentato un campanello d'allarme prezioso: proseguire con questo modello di sviluppo senza il progressivo e irreversibile danno dell'intero ecosistema, con le inevitabili drammatiche conseguenze per la nostra salute, è impensabile. Starà a noi far ricordare il 2021 come l'anno della svolta, e noi di GCND per il pianeta cercheremo di fare la nostra parte insieme a voi.

\section{Bibliografia}

1. Buizza R. Addressing climate change is a 'possible mission'. G Clin Nefrol Dial 2020;32:154-160. Online

2. Franco Bergesio. Survey report 30 June 2020 . Online

3. Online

4. Polillo G. Globalizzazione, cosa certifica l'annus horribilis 2020. Online

5. Ragusa A, Svelato A, Santacroce C, Catalano P, et al. Plasticenta: First evidence of microplastics in human placenta. Environment International, 2021, 146, Jan 2021, 106274. Online

6. Elhacham E, Ben-Uri L, Grozovski J, Bar-On YM, Milo R. Global human-made mass exceeds all living biomass. Nature 2020; 588:442-4. Online

7. Bar-On YM, Phillips R, Milo R. The biomass distribution on Earth. PNAS 2018;115(25):6506-11. Online 